\title{
Antimicrobial Activity and Chemical Composition of Momordica Charantia: A Review
}

\author{
Víctor Eduardo Villarreal-La Torre ${ }^{1, *}$, William Sagástegui Guarniz', Carmen Silva-Correa ${ }^{1}$, Lizardo Cruzado- \\ Razco', Raúl Siche ${ }^{2}$
}

\section{Víctor Eduardo Villarreal-La Torre ${ }^{1, *}$, William Sagástegui Guarniz', Carmen Silva-Correa', Lizardo Cruzado-Razco', Raúl Siche $^{2}$}

'Departamento de Farmacología, Facultad de Farmacia y Bioquímica, Universidad Nacional de Trujillo, PERU.

${ }^{2}$ Facultad de Ciencias Agropecuarias,

Universidad Nacional de Trujillo, PERU.

\section{Correspondence}

\section{Víctor Eduardo Villarreal La Torre}

Departamento de Farmacología, Facultad de Farmacia y Bioquímica, Universidad

Nacional de Trujillo, PERU

E-mail: vvillarreal@unitru.edu.pe

\section{History}

- Submission Date: 16-11-2019;

- Review completed: 9-12-2019;

- Accepted Date: 11-12-2019.

DOI : 10.5530/pj.2020.12.32

Article Available online http://www.phcogj.com/v12/i1

Copyright

(C) 2020 Phcogj.Com. This is an openaccess article distributed under the terms of the Creative Commons Attribution 4.0 International license.

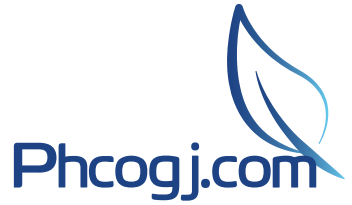

\begin{abstract}
Momordica charantia L. (bitter melon) is a plant belonging to the Cucurbitaceae family and is widely distributed in tropical and subtropical areas around the world, mainly in Asia, India, China and Brazil, where it is traditionally used as a medicinal plant, and the fruits of some varieties of $M$. charantia are consumed as food. Studies have determined that this plant contains a great diversity of bioactive compounds with therapeutic potential like charantin, $\alpha$-momorcharin and MAP30, and highlighting its properties as antidiabetic, antiulcer, antioxidant, antimicrobial, anthelmintic, antihyperglycemic and anticancer. Review shows the complete botanical description of the plant (fruits, leaves, stem, etc.), the bioactive chemical compounds reported in the plant species, the antimicrobial activity of the extracts or fractions of $M$. charantia, emphasizing the antibacterial and antifungal activities, with respective values of MIC (Minimum Inhibitory Concentration) reported according to the methodology used in each study. The review seeks to update the phytochemical and pharmacological knowledge of $M$. charantia, which would be useful for researchers in their search for new chemical compounds of the plant, studies of its safety and efficacy, as well as the evaluation of its possible synergistic action in combination with other antimicrobials, in order to find new therapeutic alternatives against bacterial resistance.
\end{abstract}

Key words: Cucurbitaceae; Phytochemicals; Antifungal; Antibacterial; Charantin; Cucurbitane.

\section{INTRODUCTION}

Plants are a very rich source of new chemical entities $^{1}$, so much so that, to date ${ }^{2}$, new prototypes with various therapeutic potentials are still being sought. ${ }^{3}$ No stranger to it, bitter melon (Momordica charantia L.) is a plant species that has attracted researchers' interest in recent years (Figure 1). Chemical and pharmacological studies on the Momordica charantia L. (M. Charantia) plant have been in existence since 1963 and have had a growing interest, deduced by the increase in the amount of research work over the years to the present; since 1993, investigations were initiated on its antibacterial activity and since 1997, on its antifungal activity (Figure 1).

The fruits of M. Charantia are consumed daily as a food and as a medicinal plant for traditional use in Southeast Asia, Indo-China ${ }^{4}$, as well as in Brazil. ${ }^{5}$ M. Charantia is a plant belonging to the Cucurbitaceae family and is widely distributed in tropical and subtropical areas around the world. ${ }^{5-7}$

Studies have determined that this plant contains a great diversity of primary and secondary metabolites ${ }^{8,9}$ with therapeutic potential as antiulcer properties $^{6,10}$, antioxidant ${ }^{11-14}$, antimicrobial ${ }^{6,8,12,15-17}$, anthelmintic ${ }^{18,19}$, antidiabetic ${ }^{6,11,20,21}$, antiinflammatory ${ }^{21,22}$, antihyperglycemic $c^{6,21,23}$ and anticancer ${ }^{4,19,21,24}$, and nutritional as antilipolytic. ${ }^{11,25}$

Bacterial resistance is one of the main problems around the world, it is thought that by 2050 bacterial resistance will be one of the leading causes of death in the world. ${ }^{26-28}$ Currently there are bacteria that are resistant to almost all existing antibacterials. ${ }^{29}$ That is why the search for new entities with antibacterial potential is a worldwide research focus ${ }^{30}$ and $M$. Charantia is a species with great possibilities. Several studies have demonstrated antifungal and antibacterial activity in $M$. Charantia $a^{9,31-34}$, as well as antimicrobial activity in leaves ${ }^{8,35-40}$, and fruit. ${ }^{41}$

In the last two years there has been a significant increase in publications of scientific articles on $M$. charantia (Figure 1), generating a large amount of information about it and its antimicrobial activity, which is why the organization and selection of this information become necessary and important in order to provide interested researchers with updated information on this species.

\section{Taxonomic classification}

M. charantia is an annual or perennial, monoclimber, herbaceous, 3-4 m long plant, which belongs to the Cucurbitaceae family. It contains almost sixty species that grow in tropical and subtropical regions (Figure 2). 4,42,43

\section{Botanical description}

Bitter melon, bitter cucumber or bitter gourd are some of the names given to M. charantia. It belongs to the Cucurbitaceae family. ${ }^{44} M$. charantia is a vegetable with many culinary uses, especially in Asia and Africa, and is commonly cultivated in Africa, India, Malaysia, China and South America. ${ }^{44,45} M$.

Cite this article: Villarreal-La Torre VE, Sagástegui Guarniz W, Silva-Correa C, Cruzado-Razco L, Siche R. Antimicrobial Activity and Chemical Composition of Momordica Charantia: A Review. Pharmacog J. 2020;12(1):213-22. 
charantia is a slender and slightly hairy or hairless plant that can be grown at high altitude. ${ }^{4,46} \mathrm{~A}$ description of each part of the $M$. charantia is shown in Table 1.

\section{Chemical composition}

M. charantia contains triterpenoids ${ }^{11,39,47}$, saponins ${ }^{48}$, polypeptides ${ }^{49}$, flavonoids ${ }^{14,50,51}$, alkaloids ${ }^{40,52,53}$ and sterols ${ }^{13,54-56}$, which are distributed

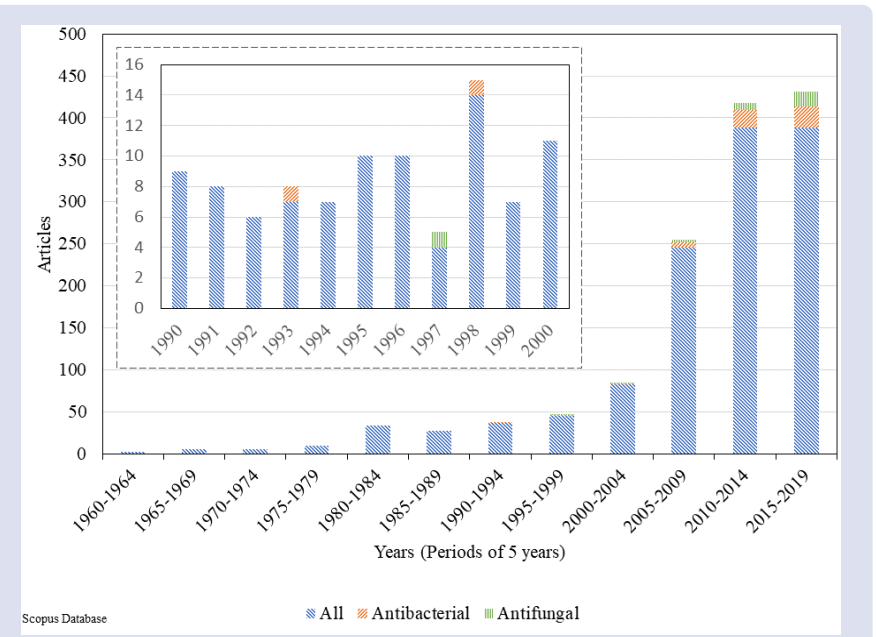

Figure 1. Number of scientific articles published on M. charantia. Search Date: 2019-04-02.

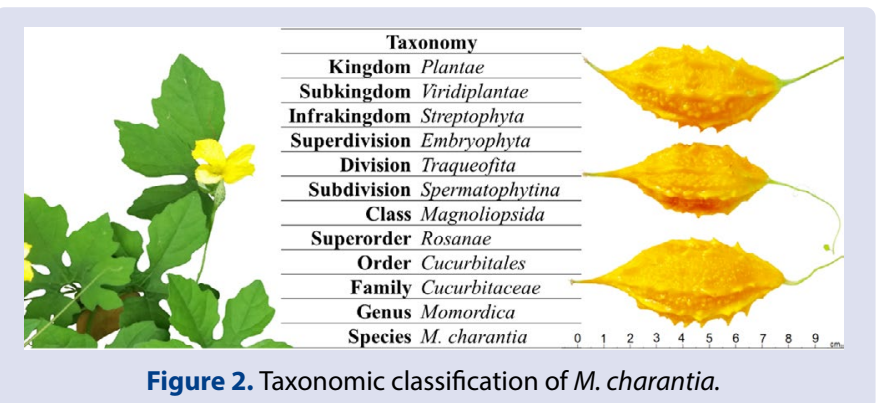

throughout the entire plant. The seed is not edible, it contains extractable oils, mostly a conjugated triene cis-9, trans-11, trans-13 $(c 9, t 11, t 13)$ conjugated isomer of linolenic acid, known as $\alpha$-elestearic acid ( $\alpha$-ESA). It is known that $\alpha$-ESA has anti-cancer and anti-obesity properties. $^{57}$

Research on $M$. charantia has revealed that its components with pharmaceutical importance are phenolic compounds (such as phenylpropanoids and flavonoids), triterpenes and carotenoids. ${ }^{50,58}$ Several bioactive compounds of the fruit of $M$. charantia have been registered in the literature; they are classified into carbohydrates, proteins, lipids and more. ${ }^{46,56,59}$

Cucurbitane-type triterpenoids such as charantin have been related to antimicrobial activity. ${ }^{60}$ Charantin is a $1: 1$ mixture of two steroidal saponins (Figure 3), stigmasterol glycoside and $\beta$-sitosterol glycoside. ${ }^{61}$ Although cucurbitane-type triterpenoids have been found in almost the entire plant, charantin has only been located in the root, leaves and fruit. $18,39,46,53$

Proteins such as $\alpha$-momorcharin (Leaf and seed) and MAP30 (fruit and seed) have also been linked to antimicrobial activity. ${ }^{62,63}$ MAP30 and a-momorcharin (Figure 4) are ribosome inactivating proteins (RIP) and have demonstrated antibacterial and antiviral activities. ${ }^{49,63}$

\section{Antimicrobial activity}

Sankaranarayanan and Jolly (1993) have clinically demonstrated the existence of antimicrobial activity on leaf extracts of $M$. charantia. This activity of $M$. charantia is attributed to its content of antimicrobial proteins, seed oil, tannins, triterpenoids, alkaloids, cardiac glycosides and steroids. ${ }^{11,46,52,64-90}$ The bioactive components of $M$. charantia showed antimicrobial activity against Helicobacter pylori ${ }^{10}$, Sindbis, Herpes simplex virus type $1^{11,82}$ and anthelmintic activity against Caenorhabditis elegans. $^{82}$

The leaf and stem extracts of $M$. charantia in methanol have a remarkable activity against Escherichia coli, Staphylococcus aureas ${ }^{83}$, Pseudomonas aeruginosa, Bacillus subtilis and Klebsiella pneumonia ${ }^{84}$, while leaf extracts in ethanol showed antimicrobial activity against Trypanosoma cruzi, in addition to enhancing the antifungal effect of metronidazole $^{85}$, E. coli, Salmonella parathyphi, Shigella dysenterae ${ }^{86}$

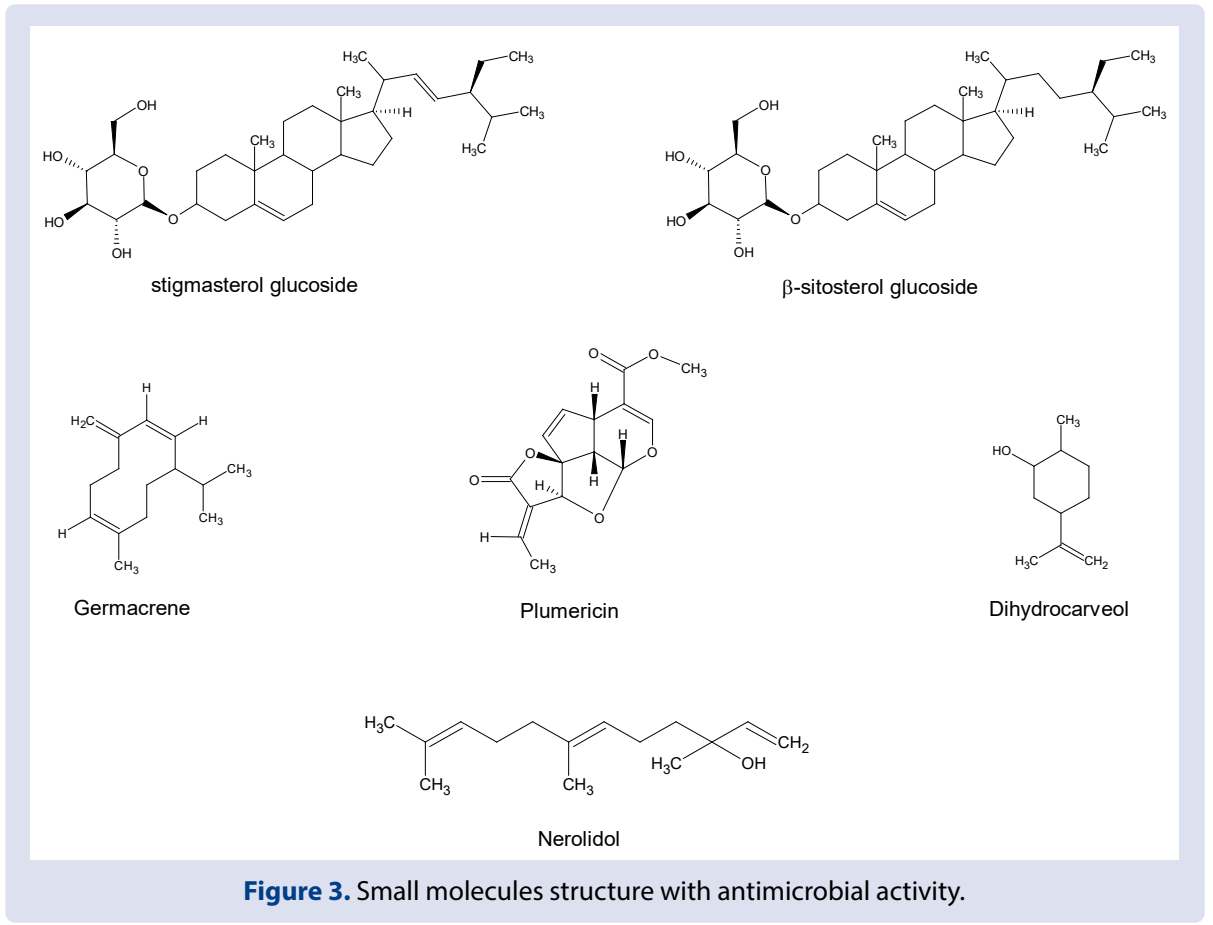




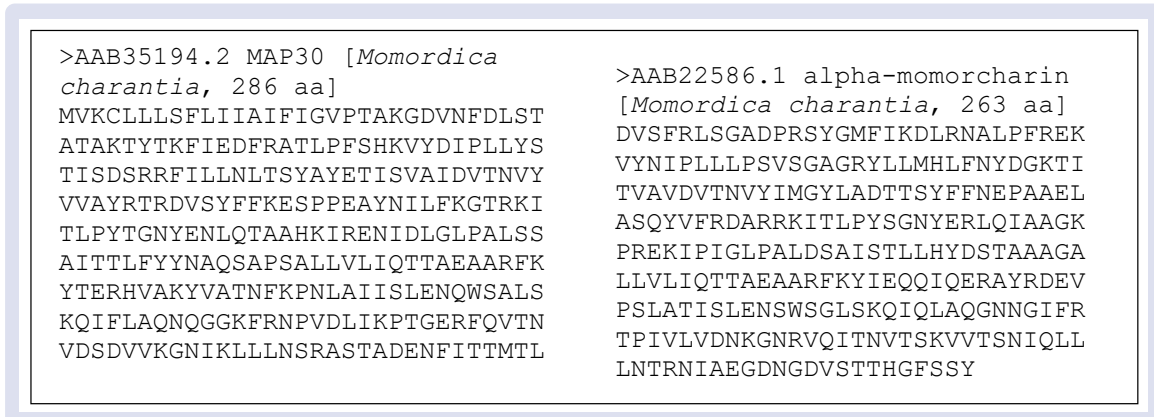

Figure 4. Amino acid sequence of MAP30 and a-momorcharin (FASTA sequence obtained from National Center for Biotechnology Information - NCBI).

\section{Table 1: Botanical description of $\boldsymbol{M}$. charantia.}

\begin{tabular}{|c|c|c|c|}
\hline Part & Description & Image & Reference \\
\hline Stem & $\begin{array}{l}\text { Round, well branched, internodes } 5-6 \mathrm{~cm} \text {, thin, corrugated and has } \\
\text { unbranched tendrils in the axillae of the leaf }\end{array}$ & & 4,46 \\
\hline Root & It has a primary root that extends to the vertex where the stem is born & & 4 \\
\hline Leaves & $\begin{array}{l}\text { Palmately-lobed, alternating, rounded edge with 3-7 lobes deeply separated } \\
\text { and with quite small marginal points. They are distributed individually in } \\
\text { petioles } 1.5-5 \mathrm{~cm} \text { long and have no stipules. When they are crushed, they give } \\
\text { off a rather unpleasant smell. }\end{array}$ & & 4,10 \\
\hline Flowers & $\begin{array}{l}\text { Solitary, pubescent and with } 5 \text { yellow petals and } 5 \text { central stamens. The male } \\
\text { flowers have thinner stems and larger petals than the female flowers and, } \\
\text { while the male flower sepals are oval-elliptical, those of the female flowers are } \\
\text { narrow and oblong lanceolate. }\end{array}$ & & 4,42 \\
\hline Fruit & $\begin{array}{l}\text { Pendular discoid with ovoid shape, } 2 \text { to } 10 \mathrm{~cm} \text { in length, covered with broken } \\
\text { or continuous longitudinal ridges and warts. The young fruit is white or } \\
\text { emerald green that turns orange when ripe, and its white pulp becomes scarlet } \\
\text { during ripening. }\end{array}$ & & $4,6,42$ \\
\hline Seed & $\begin{array}{l}8-15 \mathrm{~mm} \text { long, rectangular squares, corrugated on the margin, sculpted on } \\
\text { both sides, but covered with a white pulp when green and red when ripe. }\end{array}$ & & $4,42,46$ \\
\hline
\end{tabular}


and Colletotrichum musae. ${ }^{87}$ An extract of the whole plant has shown antiprotozoal activity against Entamoeba histolytica ${ }^{88}$, Salmonella typhi, Staphylococcus aureus, Streptococcus pyogenes ${ }^{16}$ and Mycobacterium tuberculosis ${ }^{86}$, and an extract of isolated proteins from leaves demonstrated an antifungal effect. ${ }^{36,89}$ MAP30 is an isolated protein of $M$. charantia that can be used in combination with chloramphenicol or erythromycin, and be beneficial in terms of reducing the side effects of antibiotics, as lower concentrations of antibiotics are required due to their antibacterial ability ${ }^{63}$ A synergistic effect has also been demonstrated between ethanolic extract and aminoglycosides, chlorpromazine, kanamycin and amikacin, indicating the participation of an efflux system in the resistance to these aminoglycosides. ${ }^{90}$ This represents a new weapon against bacterial resistance to antibiotics. In addition, silver nanoparticles have been studied with $M$. charantia ${ }^{52,91-93}$ although studies are still lacking to determine the real biochemical route by which it exerts its antimicrobial effects.

The leaf extract of $M$. charantia has a potent antimicrobial action against $S$. typhi with potential for hepato-inflammatory improvement by decreasing the concentrations of total and direct bilirubin, alanine aminotransferase, alkaline phosphatase, aspartate aminotransferase and gamma glutamyl transferase compared to the group control. ${ }^{38}$ In addition, photosynthetic pigments and cinnamic acid showed a direct correlation with the antimicrobial potential against Staphylococcus aureus and Pseudomonas aeruginosa, sinaptic acid showed a positive correlation only with Staphylococcus aureus; likewise, cinnamic acid, coumaric acid, syringic acid and quercetin in direct correlation with Pseudomonas aeruginosa. ${ }^{8}$ Plumericin (Figure 3), an iridoid lactone isolated from the stem of $M$. charantia, has shown antibacterial activity against Enterococcus faecalis and Bacillus subtilis with minimal inhibitory concentration values better than cloxacillin. ${ }^{66}$

The aqueous seed extract has shown greater antimicrobial capacity by inhibiting the growth of Fusarium solani ${ }^{19}$ and Pasteurella multocida, compared to the extracts of methanolic, ethanolic, hexane and ethyl acetate that were effective against $S$. aureus, Enterococcus and fungi. ${ }^{33,94-96}$ Seed oil, with $t$-nerolidol, $c$-dihydrocarveol and germacrene (Figure 3 ) as its main constituents, has shown antimicrobial activity towards S. aureus, E. coli and C. albicans ${ }^{75,97}$, which makes the development of green antibacterial soaps, without chemical aggregates, feasible. ${ }^{15}$
The levels of flavonoids and phenols such as catechin, myricetin, quercetin, gallic acid, chlorogenic acid, gentisic acid and salicylic acid, increase considerably in hair roots in vitro growth compared to unprocessed roots, although metabolites such as ferulic acid, rutin, naringenin and naringin decreased significantly in the hair roots. Due to these metabolic variations, antimicrobial activity increases in hair roots in vitro growth compared to non-transformed roots. ${ }^{51}$

Fresh fruits extracts have exhibited similar antibacterial properties against strains of Bacillus subtilis, Pseudomonas aeruginosa and Saccharomyces cerevisiae ${ }^{60}$, also have shown activity against E. coli, Staphylococcus, Pseudomonas, Salmonella and Streptobacillus very similar to the hydrophilic extracts of leaves ${ }^{6}$ and Aspergillus Niger. ${ }^{32}$ Application of $M$. charantia fruit powder at wound sites is equally effective in stimulating tissue regeneration and wound healing in rats. ${ }^{98}$ Fruit extracts have shown a better activity compared to leaf extracts ${ }^{41}$ and seeds ${ }^{40}$, with methanol extracts having the best antibacterial activity. ${ }^{37}$

Recombinant $\alpha$-momorcharin inhibits the growth of $F$. solani, causing deformation of cells with irregular outbreaks, integrity loss of the cell wall, rupture of the fungal cell membrane, DNA fragmentation, in addition to affecting macromolecular synthesis and organelles functions. ${ }^{49}$ RK29, the active lectin isolated from ripe fruit and seed, inhibits HIV-1 viral reverse transcriptase. ${ }^{6}$ Cucurbitane triterpenoids (kuguacins F-S), pentanorcucurbitacins, octanorcucurbitacin and trinorcucurbitacins exhibit weak in vitro anti-HIV-1 activities. ${ }^{99}$ The triterpene glycosides momordicines I and II are anthelmintic but not antiviral (Table 2). ${ }^{82}$

\section{Current and future challenges}

There is a growing interest in investigating the antimicrobial activity of $M$. charantia (Figure 1) motivated by the search for new sources of chemical entities with therapeutic potential. Antimicrobial activity has been reported in isolation from fruits (Table 3$)^{100}$, so it is recommended to conduct studies of the efficacy of isolated cucurbitane, such as charantin found in almost all parts of the plant (Table 2), in vivo. In addition, cucurbitane are attributed antidiabetic activity ${ }^{46}$, an effect that could enhance treatments against infections in diabetic foot.

\section{Table 2. Bioactive chemical compounds reported in $\boldsymbol{M}$. charantia.}

\begin{tabular}{|c|c|c|c|}
\hline Part of Plant & Kind of Compound & Bioactive Compounds & References \\
\hline \multirow{3}{*}{ Root } & Flavonoids & Myricetin; Quercetin; Kaempferol; Catechin; Rutin & 51 \\
\hline & Phenolic compounds & $\begin{array}{l}\text { Caffeic acid; p-Coumaric acid; Ferulic acid; o-Coumaric acid; Chlorogenic acid; } \\
\text { m-Coumaric acid; p-hydroxybenzoic acid; Gallic acid; Protocatechuic acid; } \beta \text {-Resorcylic } \\
\text { acid; Vanillic acid; Syringic acid; Gentisic acid. Salicylic acid; Vanillin; Veratric acid; } \\
\text { Hesperidin; Naringenin; Biochanin A; Homogentisic acid; } t \text {-cinnamic acid; Naringin }\end{array}$ & 51 \\
\hline & $\begin{array}{l}\text { Cucurbitane-type } \\
\text { triterpenoids }\end{array}$ & $\begin{array}{l}\text { Charantin*; kuguacins A; kuguacins B; kuguacins C; kuguacins D; kuguacins } \\
\text { E; } 3 \beta, 7 \beta, 25 \text {-trihydroxycucurbita-5,(23E)-diene-19-al; } 3 \beta, 25 \text {-dihydroxy-5 } \beta, 19 \text { - } \\
\text { epoxycucurbita-6,(23E)-diene; Momordicine I }\end{array}$ & 18,47 \\
\hline \multirow{7}{*}{$\begin{array}{l}\text { Leaf and } \\
\text { Stem }\end{array}$} & Phenolic compounds & $\begin{array}{l}\text { 4-Hydroxybenzoic acid; 4-O-Caffeoylquinic acid derivative; 4-O-Feruloylquinic acid; } \\
\text { 5-O-Feruoylquinic acid; Caffeic acid; Cinnamic acid; Ferulic acid; p-Coumaric acid; } \\
\text { sinapinic acid; 2,4-bis (2-phenylpropan-2-yl) phenol }\end{array}$ & $8,14,39,50$ \\
\hline & Flavonoids & $\begin{array}{l}\text { Isorhamnetin-3-O-glucoside; Isorhamnetin-O-acetylhexoside; Kaempferol-3-O- } \\
\text { glucoside; Kaempferol-3-O-rutinoside; Kaempferol-O-acetylhexoside; Kaempferol-O- } \\
\text { pentosylhexoside; Quercetin-3-O-glucoside; Quercetin-3-O-rutinoside; Quercetin-O- } \\
\text { acetylhexoside; Quercetin-O-dihexoside; Quercetin-O-pentosylhexoside; Rutin }\end{array}$ & $8,13,14,50,64$ \\
\hline & $\begin{array}{l}\text { Cucurbitane-type } \\
\text { triterpenoids }\end{array}$ & $\begin{array}{l}\text { Cucurbitane I; Cucurbitane II; Cucurbitane III; Karavilagenin F; Karaviloside XII; } \\
\text { Karaviloside XIII; Kuguacin F-S; Momordicine I; Momordicine II; Momordicine VI; } \\
\text { Momordicine VII; Momordicine VIII; Momordicosides; Charantal; Charantin* }\end{array}$ & $11,18,39,64,65$ \\
\hline & Iridoid lactone & Plumericin ${ }^{*}$ & 66 \\
\hline & Tannins & Not Identified & 52 \\
\hline & Alkaloids & Not Identified & 52 \\
\hline & Protein & a-momorcharin* & 49 \\
\hline
\end{tabular}




\begin{tabular}{|c|c|c|c|}
\hline \multirow[t]{2}{*}{ Flower } & Phenolic compounds & $\begin{array}{l}\text { 4-Hydroxybenzoic acid; Caffeic acid; Catechin hydrate; Chlorogenic acid; Epicatechin; } \\
\text { Ferulic acid; Gallic acid; p-Coumaric acid; } t \text {-Cinnamic acid }\end{array}$ & 50 \\
\hline & Flavonoids & Kaempferol; Rutin & 50 \\
\hline \multirow[t]{7}{*}{ Fuit } & $\begin{array}{l}\text { Cucurbitane-type } \\
\text { triterpenoids }\end{array}$ & 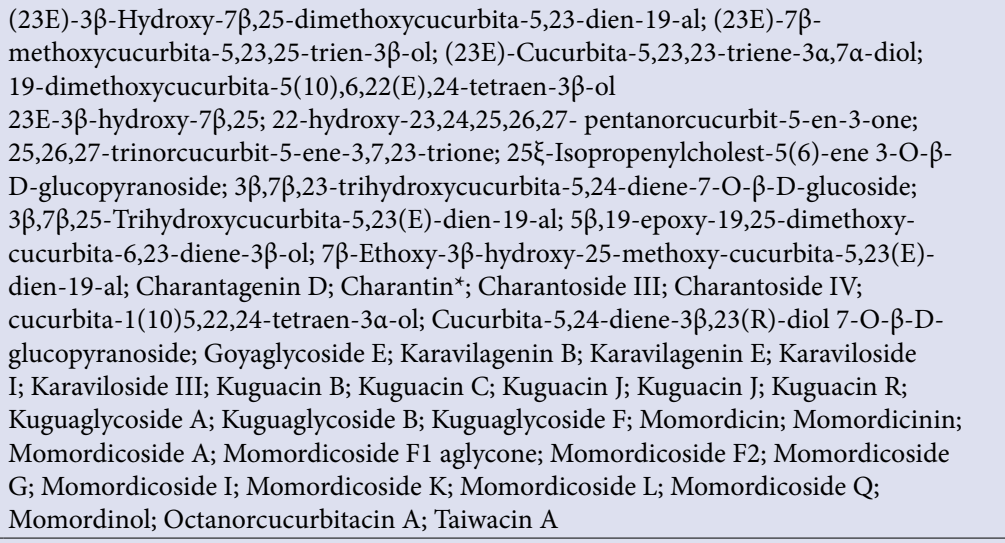 & $45,46,53,60,67-69$ \\
\hline & Carotenoids & $\begin{array}{l}\text { 5,6-Monoepoxy- } \beta \text {-Carotene; 9'-Z- neoxanthin; all-E-violaxanthin; Cryptoxanthin; } \\
\text { Lutein; Lycopene; Mutatochrome; Phytofluene; Rubixanthin; Zeaxanthin; Zeinoxanthin; } \\
\beta \text {-Carotene; } \alpha \text {-Carotene; } \gamma \text {-Carotene; } \delta \text {-Carotene; } \zeta \text {-Carotene; } \alpha \text {-tocopherol }\end{array}$ & $56,70,71$ \\
\hline & Carbohydrates & Arabinose; Galactose; Glucose; Mannose; Pectin; Rhamnose; Ribose; Xylose & 56,72 \\
\hline & Protein & MAP30 ${ }^{\text {b*}}$, Polypeptide-P, Lectins & 6,46 \\
\hline & Phytosterols & $\begin{array}{l}\text { Diosgenin; } \beta \text {-sitosterol; Stigmasterol; Campesterol; 3-O-[6'-O-stearyl- } \beta \text {-D-glucosyl]- } \\
\text { stigmasta-5,25(27)-diene; 3-O-[6'-O-palmitoyl- } \beta \text {-D-glucosyl-stigmasta-5,25(27)-dien }\end{array}$ & $13,54-56$ \\
\hline & Phenolic compounds & $\begin{array}{l}\text { Caffeic Acid; Chlorogenic acid; Ferulic acid; Gallic acid; Malic acid; Malonic Acid; } \\
\text { Quinic acid; Salicylic acid; Shikimic acid; Tartaric acid; } t \text {-cinnamic acid; Vanyl acid; } 2 \text {, } \\
\text { 5-dihydroxybenzoic acid }\end{array}$ & 45,73 \\
\hline & Alkaloids & Not Identified & 53 \\
\hline \multirow{9}{*}{ Seed } & Fatty acids & Gamolenic Acid; Linoleic acid; Oleic acid; Palmitic acid; Stearic acid; $\alpha$-Eleostearic acid & $15,57,74$ \\
\hline & Essential oils & $\begin{array}{l}\text { (E)-Anethole; } 1,8 \text {-Cineole; Apiole; Carvone; Cedrol; } c \text {-Dihydrocarveol; Germacrene } \\
\text { D; Limonene; Linalool; Methyl eugenol; Myristecin; Octanal; p-Cymene; Safrole; } \\
\text { Spathulenol; } t \text {-Dihydrocarveol; } t \text {-Nerolidol; } \alpha \text {-Pinene; } \alpha \text {-Selinene; } \beta \text {-Bisabolol; } \\
\beta \text {-Phellandrene; } \beta \text {-Pinene; } \beta \text {-Selinene; } \delta \text {-Cadinene }\end{array}$ & 75 \\
\hline & Carotenoids & Tocopherols & 56 \\
\hline & Phenolic compounds & $\begin{array}{l}\text { Caffeic acid; Catechin; Chlorogenic acid; Epicatechin; Gallic acid; Gentisic acid; } \\
\text { o-Coumaric acid; p-Coumaric acid; Protocatechuic acid; Sinapic acid; Syringic acid; } \\
t \text {-Cinnamic acid; } t \text {-Ferulic acid; Vanillic acid }\end{array}$ & 50,76 \\
\hline & $\begin{array}{l}\text { Cucurbitane-type } \\
\text { triterpenoids }\end{array}$ & $\begin{array}{l}\text { Goyaglycoside E; Goyaglycoside G; Momordicilin; Momordicoside A; Momordicoside B; } \\
\text { Momordicoside C; Momordicoside D, Momordicoside E }\end{array}$ & $46,48,77,78$ \\
\hline & Saponins & $\begin{array}{l}\text { Goyaglycoside A; Goyaglycoside B; Goyaglycoside C; Goyaglycoside D; momordicoside } \\
\text { F2; momordicoside I; momordicoside K }\end{array}$ & 48 \\
\hline & Alkaloids & Vicine & 40,46 \\
\hline & Phytosterols & $\begin{array}{l}\text { 4- } \alpha \text { - methylzymosterol; cycloeucalenol; desmethylsterols spinasterol; lophenol; } \\
\text { obtusifoliol; } \beta \text {-sitosterol }\end{array}$ & 11,79 \\
\hline & Protein & $\begin{array}{l}\text { Cytostatic factor; } \mathrm{MCL}^{\mathrm{a}} \text {; MAP30 } \text {; Momordin-I; Momordin-II; Napin-like } \\
\text { protein; Napin-like RIP; Polypeptide-P; Ribonuclease; Serpins; } \alpha \text {-momorcharin }{ }^{*} \text {; } \\
\beta \text {-momorcharin; } \gamma \text {-momorcharin; Polypeptide-P; Lectins }\end{array}$ & $53,80,81$ \\
\hline
\end{tabular}

${ }^{\mathrm{a}} \mathrm{MCL} M$. charantia lectin ${ }^{\mathrm{b}} \mathrm{MAP} 30$ a $30 \mathrm{kDa}$ M. charantia anti-HIV protein; ${ }^{\mathrm{C}} \mathrm{RIP}$ ribosome inactivating protein; ${ }^{*}$ relevant for antimicrobial activity.

Table 3. Antimicrobial activity of extracts or fractions of $M$. charantia.

\begin{tabular}{|c|c|c|c|c|c|}
\hline Part & Extract or Fraction & Activity & MIC & Technique & Reference \\
\hline \multirow{13}{*}{ leaf } & \multirow{6}{*}{ Methanolic extract } & Escherichia coli & \multirow{2}{*}{$10 \mathrm{mg} / \mathrm{mL}$} & \multirow{2}{*}{ Agar cup well technique } & \multirow{2}{*}{83} \\
\hline & & Staphylococcus aureus & & & \\
\hline & & Escherichia coli & \multirow{4}{*}{$100 \mathrm{mg} / \mathrm{mL}$} & \multirow{4}{*}{ disc diffusion method } & \multirow{4}{*}{84} \\
\hline & & Pseudomonas aeruginosa & & & \\
\hline & & Bacillus subtilis & & & \\
\hline & & Klebsiella pneumonia & & & \\
\hline & Ethanolic extract & Staphylococcus aureus & $125 \mu \mathrm{g} / \mathrm{mL}$ & microdilution & 64 \\
\hline & \multirow{4}{*}{ Aqueous Extract } & Escherichia coli & \multirow{4}{*}{$100 \mathrm{mg} / \mathrm{mL}$} & \multirow{4}{*}{ disc diffusion method } & \multirow{4}{*}{84} \\
\hline & & Pseudomonas aeruginosa & & & \\
\hline & & Bacillus subtilis & & & \\
\hline & & Klebsiella pneumonia & & & \\
\hline & \multirow{2}{*}{ Acetone Extract } & Pseudomonas aeruginosa & $2 \mu \mathrm{g} / \mathrm{mL}$ & \multirow{2}{*}{ microdilution } & \multirow{2}{*}{8} \\
\hline & & Staphylococcus aureus & $3 \mu \mathrm{g} / \mathrm{mL}$ & & \\
\hline
\end{tabular}




\begin{tabular}{|c|c|c|c|c|c|}
\hline \multirow[b]{2}{*}{ Stem } & \multirow{2}{*}{$\begin{array}{l}\text { Plumericin Isolated using } \\
\text { the supercritical fluid } \\
\text { extraction }\end{array}$} & Enterococcus faecalis & $250 \mu \mathrm{g} / \mathrm{mL}$ & \multirow[b]{2}{*}{ disc diffusion method } & \multirow[b]{2}{*}{66} \\
\hline & & Bacillus suptilis & $125 \mu \mathrm{g} / \mathrm{mL}$ & & \\
\hline \multirow{11}{*}{ Fruit } & \multirow{3}{*}{ Charantin } & Bacillus suptilis & \multirow{3}{*}{$200 \mu \mathrm{g} / \mathrm{mL}$} & \multirow{3}{*}{ disc diffusion method } & \multirow{3}{*}{60} \\
\hline & & Pseudomonas aeruginosa & & & \\
\hline & & Saccharomyces cerevisiae & & & \\
\hline & \multirow{3}{*}{ Ethanolic extract } & Aspergillus niger & & \multirow{3}{*}{ disc diffusion method } & \multirow{3}{*}{32} \\
\hline & & Staphylococcus aureus & Not determined & & \\
\hline & & Salmonella typhi & & & \\
\hline & \multirow{3}{*}{ Chloroform extract } & Escherichia coli & $200 \mu \mathrm{g} / \mathrm{mL}$ & \multirow{5}{*}{ microdilution } & \multirow{5}{*}{100} \\
\hline & & Bacillus suptilis & $200 \mu \mathrm{g} / \mathrm{mL}$ & & \\
\hline & & Aspergillus niger & $100 \mu \mathrm{g} / \mathrm{mL}$ & & \\
\hline & Hexane extract & \multirow{2}{*}{ Aspergillus niger } & \multirow{2}{*}{$100 \mu \mathrm{g} / \mathrm{mL}$} & & \\
\hline & Ethyl acetate extract & & & & \\
\hline \multirow{16}{*}{ Seed } & Extraction with buffer & Fusarium solani & $108.9 \mu \mathrm{g} / \mathrm{mL}$ & well diffusion and broth & 19 \\
\hline & $\begin{array}{l}\text { Methanolic extraction by } \\
\text { soxhlet }\end{array}$ & Enterococcus faecium & $20 \mathrm{~mm}$ & disc diffusion method & 96 \\
\hline & \multirow{11}{*}{ Ethanolic extract } & Proteus mirabilis & $3 \mu \mathrm{g} / \mathrm{mL}$ & \multirow{11}{*}{ microdilution } & \multirow{11}{*}{33} \\
\hline & & Providencia rettgeri & $7 \mu \mathrm{g} / \mathrm{mL}$ & & \\
\hline & & Staphylococcus aureus & $31 \mu \mathrm{g} / \mathrm{mL}$ & & \\
\hline & & Escherichia coli & $31 \mu \mathrm{g} / \mathrm{mL}$ & & \\
\hline & & Pseudomonas aeruginosa & $62 \mu \mathrm{g} / \mathrm{mL}$ & & \\
\hline & & Candida parapsilosis & $15 \mu \mathrm{g} / \mathrm{mL}$ & & \\
\hline & & Candida guillermond & $31 \mu \mathrm{g} / \mathrm{mL}$ & & \\
\hline & & Candida glabata & $31 \mu \mathrm{g} / \mathrm{mL}$ & & \\
\hline & & Candida tropicallis & $62 \mu \mathrm{g} / \mathrm{mL}$ & & \\
\hline & & Candida albicans & $62 \mu \mathrm{g} / \mathrm{mL}$ & & \\
\hline & & Candida krusei & $125 \mu \mathrm{g} / \mathrm{mL}$ & & \\
\hline & \multirow{3}{*}{ Hydrodistillation } & Sthaphylococcus aureus & $125 \mu \mathrm{g} / \mathrm{mL}$ & \multirow{3}{*}{ microdilution } & \multirow{3}{*}{75} \\
\hline & & Escherichia coli & $>500 \mu \mathrm{g} / \mathrm{mL}$ & & \\
\hline & & Candida albicans & $>500 \mu \mathrm{g} / \mathrm{mL}$ & & \\
\hline \multirow{3}{*}{ Whole plant } & Fraction 1 & Salmonella typhi & $40 \mu \mathrm{g} / \mathrm{mL}$ & \multirow{3}{*}{ Agar cup well technique } & \multirow{3}{*}{16} \\
\hline & Fraction 2 & $\begin{array}{l}\text { Staphylococcus aureus } \\
\text { Streptococcus pyogenes }\end{array}$ & $60 \mu \mathrm{g} / \mathrm{mL}$ & & \\
\hline & Fraction 3 & Streptococcus pyogenes & $40 \mu \mathrm{g} / \mathrm{mL}$ & & \\
\hline
\end{tabular}

On the other hand, studies of its safety and efficacy have been carried out in combination with antimicrobials such as aminoglycosides ${ }^{90}$, with the intention of being able to cope with bacterial resistance as well as a decrease in side effects; Therefore, it is recommended to continue studies on proteins such as a-momorcharin and MAP30 which is located in leaves, stems, fruits and seeds (Table 2), which have demonstrated very good antimicrobial metabolites ${ }^{49,63}$ as well as some protein fractions ${ }^{89}$. Although there are a large number of articles that corroborate the antimicrobial activity, the mechanism of this therapeutic activity is not yet known.

\section{CONCLUSIONS}

Although a large number of medicinal plants have been reported with antimicrobial activity, studies that corroborate their efficacy and safety are still needed. The phytochemical analysis and demonstration of the in vivo and in vitro antimicrobial activity of $M$. charantia, promotes the need to study the probable mechanisms by which bioactive compounds such as charantin, $\alpha$-momorcharin and MAP30 act.

\section{REFERENCES}

1. Salim AA, Chin YW, Kinghorn AD. Drug Discovery from Plants. In: Bioactive Molecules and Medicinal Plants. Berlin, Heidelberg: Springer; 2008. p. 1-24.

2. Vambe M, Aremu AO, Chukwujekwu JC, Finnie JF, Van SJ. Antibacterial screening, synergy studies and phenolic content of seven South African medicinal plants against drug-sensitive and -resistant microbial strains. S Afr J Bot. 2018;114:250-9.
3. Mumtaz A, Ashfaq UA, Tahir M, Gulzar F, Ali MA, Saari N, et al. MPD3: a useful medicinal plants database for drug designing. Nat Prod Res. 2016;31(11):122836.

4. Poolperm S, Jiraungkoorskul W. An update review on the anthelmintic activity of bitter gourd, Momordica charantia. Pharmacogn Rev. 2017;11(21):31-4.

5. Magalhães KDN, Guarniz WAS, Sá KM, Freire AB, Monteiro MP, Nojosa RT, et al. Medicinal plants of the Caatinga, northeastern Brazil: Ethnopharmacopeia (1980-1990) of the late professor Francisco José de Abreu Matos. J Ethnopharmacol. 2019;237:314-53.

6. Jia S, Shen M, Zhang F, Xie J. Recent advances in Momordica charantia: Functional components and biological activities. Int J Mol Sci. 2017;18(12):1-25.

7. Basch E, Gabardi S, Ulbricht C. Bitter melon (Momordica charantia): A review of efficacy and safety. Am J Heal Sys.t Pharm. 2003;60(4):356-359.

8. Bukhari SA, Farah N, Mustafa G, Mahmood S, Naqvi SAR. Magneto-priming improved nutraceutical potential and antimicrobial activity of Momordica charantia L. without affecting nutritive value. Appl Biochem Biotechnol. 2019;188(3):878-892

9. Supe U, Daniel P. HPLC method for analysis of bioactive compound from Momordica charantia. Am J Agric Environ Sci. 2015;15(11):2196-200.

10. Gupta M, Sharma S, Gautam A, Bhaduria R. Momordica charantia Linn (Karela): Nature's silent healer. Int J Pharm Sci Rev Res. 2011;11(1):32-7.

11. Wang S, Li Z, Yang G, Ho CT, Li S. Momordica charantia: A popular healthpromoting vegetable with multifunctionality. Food Funct. 2017;8(5):1749-62.

12. Shafie MH, Samsudin D, Yusof R, Gan C-Y. Characterization of bio-based plastic made from a mixture of Momordica charantia bioactive polysaccharide and choline chloride/glycerol based deep eutectic solvent. Int J Biol Macromol. 2018;118(Part A):1183-92.

13. Mala M, Hepsibah AH, Jothi GJ. Silver nanoparticles synthesis using Coccinia Grandis (L.) voigt and Momordica Charantia L, its characterization and biological screening. J Bionanosci. 2017;11(6):504-13. 
14. Svobodova B, Barros L, Calhelha RC, Heleno S, Alves MJ, Walcott S, et al. Bioactive properties and phenolic profile of Momordica charantia L. medicinal plant growing wild in Trinidad and Tobago. Ind Crop Prod. 2017;95:365-73.

15. Zubair MF, Atolani O, Ibrahim SO, Oguntoye OS, Abdulrahim HA, Oyegoke RA, et al. Chemical and biological evaluations of potent antiseptic cosmetic products obtained from Momordica charantia seed oil. Sustain Chem Pharm. 2018;9:35-41.

16. Abalaka ME, Onaolapo JA, Inabo HI, Olonitola OS. Antibacterial activity of chromatographically separated pure fractions of whole plant of Momordica charantia L (Cucurbitaceae). Adv Environ Biol. 2010;4(3):509-14.

17. Abid M, Chohan S, Mehmood MA, Naz S, Naqvi SAH. Antifungal potential of indigenous medicinal plants against Myrothecium leaf spot of bitter gourd (Momordica charantia L.). Braz arch biol technol. 2018;60:e17160395.

18. Swarna J, Ravindhran R. Agrobacterium rhizogenes - mediated hairy root induction of Momordica charantia Linn. and the detection of charantin, a potent hypoglycaemic agent in hairy roots. Res J Biotechnol. 2012;7(4):227-31.

19. Wang S, Zheng Y, Xiang F, Li S, Yang G. Antifungal activity of Momordica charantia seed extracts toward the pathogenic fungus Fusarium solani L. J Food Drug Anal. 2016;24(4):881-7.

20. Peter EL, Kasali FM, Deyno S, Mtewa A, Nagendrappa PB, Tolo CU, et al. Momordica charantia L. lowers elevated glycaemia in type 2 diabetes mellitus patients: Systematic review and meta-analysis. J Ethnopharmacol. 2018;231:311-24.

21. Lucas EA, Dumancas GG, Smith BJ, Clarke SL, Arjmandi BH. Health benefits of bitter melon (Momordica charantia). In: Watson RR, Preedy VR, editors Bioactive Foods in Promoting Health. San Diego: Academic Press;2010. p. 52549.

22. Zeng Y, Guan M, Li C, Xu L, Zheng Z, Li J, et al. Bitter melon (Momordica charantia) attenuates atherosclerosis in apo-E knock-out mice possibly through reducing triglyceride and anti-inflammation. Lipids Heal Dis. 2018;17(1):251.

23. Palamthodi S, Lele SS. Nutraceutical applications of gourd family vegetables: Benincasa hispida, Lagenaria siceraria and Momordica charantia. Biomed Prev Nutr. 2014;4(1):15-21.

24. Ji H, Zhang L, Li J, Yang M, Liu X. Optimization of ultrahigh pressure extraction of momordicosides from bitter melon. Int J Food Eng. 2010;6(6):Article 3.

25. Nerurkar P V., Lee YK, Nerurkar VR. Momordica charantia (bitter melon) inhibits primary human adipocyte differentiation by modulating adipogenic genes. BMC Complement Altern Med. 2010;10:34

26. de Kraker M, Stewardson A, Harbarth S. Will 10 Million People Die a Year due to Antimicrobial Resistance by 2050? PLoS Med. 2016;13(11):1-6.

27. Sanchez LO, Gustot T. Multidrug-resistant bacterial infection in patients with cirrhosis. A review. Curr Hepatol Rep. 2019;18(1):28-35.

28. Boklage $E$, Lehmkuhl M. Coverage of antimicrobial resistance in the German Press: 1993-2013. Heal Commun. 2018;34(9):958-63.

29. McCarthy M. Woman dies after infection with bacteria resistant to all antibiotics available in US. BMJ. 2017;356:j254.

30. Tacconelli E, Carrara E, Savoldi A, Harbarth S, Mendelson M, Monnet DL, et al. Discovery, research, and development of new antibiotics: the WHO priority list of antibiotic-resistant bacteria and tuberculosis. Lancet Infect Dis. 2018;18(3):318-27.

31. Adeyemi AO, Adeyinka AV, Olawande FT. Antibacterial activities of aqueous extracts of Terminalia catappa, Momordica charantia and Acaiypha wilkesiana on Escherichia coli isolated from pediatrics. Pak j sci ind res Ser B biol sci. 2015;58(2):72-6.

32. Yaldiz G, Sekeroglu N, Kulak M, Demirkol G. Antimicrobial activity and agricultural properties of bitter melon (Momordica charantia L.) grown in northern parts of Turkey: A case study for adaptation. Nat Prod Res. 2015;29(6):543-5.

33. Lucena Filho JH, Lima Rde F, Medeiros AC, Pereira JV, Granville-Garcia AF, Costa EM. Antimicrobial potential of Momordica charantia L. against multiresistant standard species and clinical isolates. J Contemp Dent Pr. 2015;16(11):854-8.

34. Karmakar M, Kumar K, Sharanagat VS, Dixit A. Green synthesis and characterization of silver nanoparticle using Momordica charantia and Manilkara zapota seeds. Eco Env Cons. 2015;21(Suppl.Issue August 2015):AS251-7.

35. Brandão DO, Guimarães GP, Santos RL, Júnior FJLR, da Silva KMA, de Souza FS, et al. Model analytical development for physical, chemical, and biological characterization of Momordica charantia vegetable drug. J Anal Methods Chem. 2016;2016:15 pages.

36. Zhang B, Xie C, Wei Y, Li J, Yang X. Purification and characterisation of an antifungal protein, MCha-Pr, from the intercellular fluid of bitter gourd (Momordica charantia) leaves. Protein Expr Purif. 2015;107:43-9.

37. Supraja P, Usha R. Antibacterial and phytochemical screening from leaf and fruit extracts of Momordica charantia. Int J Pharm Bio Sci. 2013;4(1):787-93.

38. Adeyi AO, Jinadu AM, Arojojoye OA, Alao OO, Ighodaro OM, Adeyi OE. In vivo and in vitro antibacterial activities of Momordica charantia on Salmonella typh and its effect on liver function in typhoid-infected rats. J Pharmacogn Phytother. 2013;5(11):183-8.
39. Panlilio BG, Macabeo APG, Knorn M, Kohls P, Richomme P, Kouam SF, et al. A lanostane aldehyde from Momordica charantia. Phytochem Lett. 2012;5(3):682

40. Makhija M, Ahuja D, Nandy BC, Gautam S, Tiwari K, Awasthi A, et al. Evaluation and comparison of antibacterial activity of leaves, seeds and fruits extract of Momordica charantia. Res J Pharm Biol Chem Sci. 2011;2(2):185-92.

41. Mwambete KD. The in vitro antimicrobial activity of fruit and leaf crude extracts of Momordica charantia: A Tanzania medicinal plant. Afr Heal Sci. 2009;9(1):34 9.

42. Bharathi LK, John KJ. Momordica genus in Asia - An overview. Vol. 9788132210 Springer. New Delhi; 2013. 1-147 p.

43. Rafaela DS, Marília BC, Rafael José RP, Luiz CA, Karina Perrelli R. Anatomical study and characterization of metabolites in leaves of Momordica charantia L. Pharmacog J. 2018;10(5):823-6.

44. Karaman K, Dalda-Sekerci A, Yetișir H, Gülșen O, Cosskun ÖF. Molecular morphological and biochemical characterization of some Turkish bitter melon (Momordica charantia L.) genotypes. Ind Crop Prod. 2018;123:93-9.

45. Perez JL, Jayaprakasha GK, Patil BS. Metabolite profiling and in vitro biological activities of two commercial bitter melon (Momordica charantia Linn.) cultivars. Food Chem. 2019;288:178-86.

46. Upadhyay A, Agrahari P, Singh DK. A review on salient pharmacological features of Momordica charantia. Int J Pharmacol. 2015;11(5):405-13.

47. Chen JC, Lu L, Zhang XM, Zhou L, Li ZR, Qiu MH. Eight new cucurbitane glycosides, kuguaglycosides A-H, from the root of Momordica charantia L. Helv Chim Acta. 2008;91(5):920-9.

48. Popovich DG, Li L, Zhang W. Bitter melon (Momordica charantia) triterpenoid extract reduces preadipocyte viability, lipid accumulation and adiponectin expression in 3T3-L1 cells. Food Chem Toxicol. 2010;48(6):1619-26.

49. Wang S, Zhang Y, Liu H, HeY, Yan J, Wu Z, et al. Molecular cloning and functional analysis of a recombinant ribosome-inactivating protein (alpha-momorcharin) from Momordica charantia. Appl Microbiol Biotechnol. 2012;96(4):939-50.

50. Cuong DM, Kwon SJ, Jeon J, Park YJ, Park JS, Park SU. Identification and characterization of phenylpropanoid biosynthetic genes and their accumulation in bitter melon (Momordica charantia). Molecules. 2018;23(2):469.

51. Thiruvengadam M, Praveen N, Maria John KM, Yang Y-S, Kim S-H, Chung I-M Establishment of Momordica charantia hairy root cultures for the production of phenolic compounds and determination of their biological activities. Plant Cell Tiss Organ Cult. 2014;118(3):545-57.

52. Ajitha B, Reddy YAK, Reddy PS. Biosynthesis of silver nanoparticles using Momordica charantia leaf broth: Evaluation of their innate antimicrobial and catalytic activities. J Photochem Photobiol B. 2015;146:1-9.

53. Joseph B, Jini D. Antidiabetic effects of Momordica charantia (bitter melon) and its medicinal potency. Asian Pac J Trop Dis. 2013;3(2):93-102.

54. Guevara AP, Lim-Sylianco CY, Dayrit FM, Finch P. Acylglucosyl sterols from Momordica charantia. Phytochemistry. 1989;28(6):1721-4.

55. Park H, Moon B, Kim S. Antioxidant and $\alpha$-glucosidase inhibitory activities of fresh bitter melon and change of charantin and lutein content upon brining and blanching treatments of pickling. Korean J Food Sci Technol. 2018;50(4):430-6.

56. Rohajatien U, Harijono H, Estiasih T, Sriwahyuni E. Bitter melon (Momordica charantia L) fruit decreased blood glucose level and improved lipid profile of streptozotocin induced hyperglycemia rats. Curr Res Nutr Food Sci. 2018;6(2):359-70.

57. Chan FK, Hsu C, Li TC, Chen WH, Tseng KT, Chao PM. Bitter melon seed oil increases mitochondrial content in gastrocnemius muscle and improves running endurance in sedentary C57BL/6J mice. J Nutr Biochem. 2018;58:1507.

58. Saeed MK, Shahzadi I, Ahmad I, Ahma R, Shahzad K, Ashraf M, et al. Nutritional analysis and antioxidant activity of bitter gourd (Momordica charantia) from Pakistan. Pharmacologyonline. 2010;1:252-60.

59. Zhang F, Lin L, Xie J. A mini-review of chemical and biological properties of polysaccharides from Momordica charantia. Int J Biol Macromol. 2016;92:24653.

60. Patel S, Patel T, Parmar K, Bhatt Y, Patel Y, Patel NM. Isolation, characterization and antimicrobial activity of charantin from Momordica Charantia Linn. fruit. Int J Drug Dev Res. 2010;2(3):629-34.

61. Desai S, Tatke P. Charantin: An important lead compound from Momordica charantia for the treatment of diabetes. J Pharmacogn Phytochem. 2015;3(6):163-6.

62. Zhu F, Zhang P, Meng YF, Xu F, Zhang DW, Cheng J, et al. Alpha-momorcharin, a RIP produced by bitter melon, enhances defense response in tobacco plants against diverse plant viruses and shows antifungal activity in vitro. Planta. 2013;237(1):77-88.

63. Ching-Dong C, Ping-Yuan L, Yo-Chia C, Han-Hsiang H, Wen-Ling S. Novel purification method and antibiotic activity of recombinant Momordica charantia MAP30. 3 Biotech. 2017;7(1):3. 
64. Guarniz W, Canuto K, Ribeiro P, Dodou H, Magalhaes K, Sa K. Momordica Charantia L. variety from Northeastern Brazil : Analysis of Antimicrobial Activity and Phytochemical Components. Pharmacog J. 2019;11(6):1312-24.

65. Zhao GT, Qiu MH, Zhou L, Zhang ZR, Li HZ, Liu JQ, et al. Cucurbitane-type triterpenoids from the stems and leaves of Momordica charantia. Fitoterapia. 2014; $95: 75-82$

66. Saengsai J, Kongtunjanphuk S, Yoswatthana N, Kummalue T, Jiratchariyakul W. Antibacterial and antiproliferative activities of plumericin, an iridoid isolated from Momordica charantia vine. Evid-Based Compl Alt. 2015;2015:10 pages.

67. Perez JL, Jayaprakasha GK, Patil BS. Separation and Identification of Cucurbitane-Type Triterpenoids from Bitter Melon. In: Jayprakasha GK, Patil BS, Pellati $F$, editors. Instrumental Methods for the Analysis and Identification of Bioactive Molecules. Texas: American Chemical Society; 2014. p. 51-78.

68. Okabe H, Miyahara Y, Yamauchi T. Studies on the constituents of Momordica charantia L. III. Characterization of new cucurbitacin glycosides of the immature fruits. (1). Structures of momordicosides G, F1, F2 and I. Chem Pharm Bull. 1982;30(11):3977-86.

69. Okabe H, Miyahara Y, Yamauchi T. Studies on the constituents of Momordica charantia L. IV. Characterization of the new cucurbitacin glycosides of the immature fruits. (2) Structures of the bitter glycosides, momordicosides K and L. Chem Pharm Bull. 1982;30(12):4334-40.

70. Rodriguez DB, Raymundo LC, Lee T-C, Simpson KL, Chichester CO. Carotenoid pigment changes in ripening Momordica charantia fruits. Ann Bot-London. 1976;40(3):615-24.

71. Saini RK, KeumYS. Characterization of nutritionally important phytoconstituents in bitter melon (Momordica charantia L.) fruits by HPLC-DAD and GC-MS. J Food Meas Charact. 2017;11(1):119-25

72. Deng YY, Yi Y, Zhang LF, Zhang RF, Zhang Y, Wei ZC, et al. Immunomodulatory Activity and Partial Characterisation of Polysaccharides from Momordica charantia. Molecules. 2014;19(9):13432-47.

73. Annapoorani CA, Manimegalai K. Evaluation of biological activity and qualitative analysis of 2,5-dihydroxybenzoic acid from Momordica charantia fruit. Int J Pharm Sci Rev Res. 2013:22(2):89-95.

74. Ghaffar F, Kainatm B, Shah HU, Rahman IU. DPPH Radical Scavenging Assay, Biological Activities, Nutricional Composition and Quality Parameters of Momordica charantia Seeds Grown in District CHarsadda, KPK, Pakistan. Pak J Sci Ind Res Ser B Biol Sci. 2017;60(2):80-6

75. Braca A, Siciliano T, D’Arrigo M, Germanò MP. Chemical composition and antimicrobial activity of Momordica charantia seed essential oil. Fitoterapia. 2008;79(2):123-5.

76. Horax R, Hettiarachchy N, Islam S. Total phenolic contents and phenolic acid constituents in 4 varieties of bitter melons (Momordica charantia) and antioxidant activities of their extracts. J Food Sci. 2005;70(4):C275-80.

77. Okabe H, Miyahara $Y$, Yamauchi T, Miyahara K, Kawasaki T. Studies on the constituents of Momordica charantia L. I. Isolation and characterization of momordicosides A and B, glycosides of a pentahydroxy-cucurbitane triterpene. Chem Pharm Bull. 1980;28(9):2753-62.

78. Miyahara Y, Okabe H, Yamauchi T. Studies on the constituents of Momordica charantia L. II. isolation and characterization of minor seed glycosides, momordicosides C, D and E. Chem Pharm Bull. 1981;29(6):1561-6.

79. Liu R, Gupta SK, Liaw S, Chyuan J-H, Tsay H-S. In vitro studies on newly developed variety of Momordica charantia L. Hualien No. 1 and its metabolites analysis. Int J Integ Biol. 2011;11(3):155-60.

80. Fang EF, Ng TB. The bitter fruit with sweet health benefits: A comprehensive synopsis of recent research progress on medicinal properties of Momordica charantia. In: Fang EF, Ng TB, editors. Antitumor Potential and other Emerging Medicinal Properties of Natural Compounds. Baltimore: Springer; 2013. p. 31534.

81. Li SS. Purification and partial characterization of two lectins from Momordica charantia. Experientia. 1980;36(5):524-7.
82. Beloin N, Gbeassor M, Akpagana K, Hudson J, de Soussa K, Koumaglo K, et al. Ethnomedicinal uses of Momordica charantia (Cucurbitaceae) in Togo and relation to its phytochemistry and biological activity. J Ethnopharmacol. 2005;96(1-2):49-55.

83. Lu YL, Liu YH, Chyuan JH, Cheng KT, Liang WL, Hou WC. Antibacterial and cytotoxic activities of different wild bitter gourd cultivars (Momordica charantia L. var. abbreviata Seringe). Bot Stud. 2011;52(4):427-34.

84. Leelaprakash G, Rose J, Gowtham B, Javvaji PK, Prasad S. In vitro Antimicrobia and Antioxidant Activity of Momordica Charantia Leaves. Pharmacophore. $2011 ; 2(4): 244-52$

85. Santos KKA, Matias EFF, Sobral-Souza CE, Tintino SR, Morais-Braga MFB Guedes GMM, et al. Trypanocide, cytotoxic, and antifungal activities of Momordica charantia. Pharm Biol. 2012;50(2):162-6.

86. Omoregbe RE, Ikuebe OM, Ihimire IG. Antimicrobial activity of some medicinal plants extracts on Escherichia coli, Salmonella paratyphi and Shigella dysenteriae. Afr J Med Med Sci. 1996 Dec;25(4):373-5

87. Celoto MIB, Papa MFS, Sacramento LVS, Celoto FJ. Atividade antifúngica de extratos de Momordica charantia L. sobre Colletotrichum musae. Rev Bras Plantas Med. 2011;13(3):337-41.

88. Sankaranarayanan J, Jolly C. Phytochemical, antibacterial and pharmacological investigations on Momordica charantia linn. Emblica officinalis gaertn. and Curcuma longa linn. Indian J Pharm Sci. 1993;55(1):6-13.

89. Qiao Y, Song L, Zhu C, Wang Q, Guo T, Yan Y, et al. Dataset on preparation of the phosphorylated counterparts of a Momordica charantia protein for studying antifungal activities against susceptible dose-dependent C. albicans to antimycotics. Data Br. 2017;15:370-5.

90. Coutinho HDM, Costa JGM, Lima EO, Falcão-Silva VS, Siqueira-Júnior JP. In vitro interference of Momordica charantia in the resistance to aminoglycosides. Pharm Biol. 2009;47(11):1056-9

91. Malaikozhundan B, Vaseeharan B, Vijayakumar S, Sudhakaran R, Gobi N, Shanthini G. Antibacterial and antibiofilm assessment of Momordica charantia fruit extract coated silver nanoparticle. Biocata.I Agric Biotechnol. 2016;8:189 96.

92. Rashid MMO, Akhter KN, Chowdhury JA, Hossen F, Hussain MS, Hossain MT. Characterization of phytoconstituents and evaluation of antimicrobial activity of silver-extract nanoparticles synthesized from Momordica charantia fruit extract. BMC Complement Altern Med. 2017;17:336

93. Gandhiraj V, Sathish Kumar K, Narendrakumar G. Biotic synthesis of silver nanoparticles from Momordica charantia (Cucurbitaceae) and its characterization studies. Res J Biotech. 2018;13(9):90-9.

94. Roopashree TS, Dang R, Shobha RRH, Narendra C. Antibacterial activity of antipsoriatic herbs: Cassia tora, Momordica charantia and Calendula officinalis. Int J Appl Res Nat Prod. 2008;1(3):20-8.

95. Mahmood A, Raja G, Mahmood T, Gulfraz M, Khanum A. Isolation and characterization of antimicrobial activity conferring component(s) from seeds of bitter gourd (Momordica charantia). J Med Plants Res. 2012;6(4):566-73.

96. Andleeb S, Ghous T, Riaz N, Shahzad N, Ghous S, Awan UA. Assessment of antibacterial activity of Momordica charantia extracts and antibiotics against fecal contaminated water associated Enterococcus spp. Pakistan J Zool 2013;45(2):555-8.

97. Jabeen $U$, Khanum A. Isolation and characterization of potential food preservative peptide from Momordica charantia L. Arab J Chem. 2017;10(2):S3982-9.

98. Prasad V, Jain V, Girish D, Dorle AK. Wound-healing property of Momordica charantia L. fruit powder. J Herb Pharmacother. 2006;6(3-4):105-15.

99. Chen J,Tian R, Qiu M, Lu L, Zheng Y, Zhang Z. Trinorcucurbitane and cucurbitane triterpenoids from the roots of Momordica charantia. Phytochemistry 2008;69(4):1043-8

100. Dam NP, Dien MV, Thanh LHV, Hien PTT, Tram NTT. Investigatión of antimicrobial activity and chemical constituents of Momordica charantia L. var. abbreviata Ser. Vietnam J Sci Technol. 2019;57(2):155-61. 


\section{GRAPHICAL ABSTRACT}

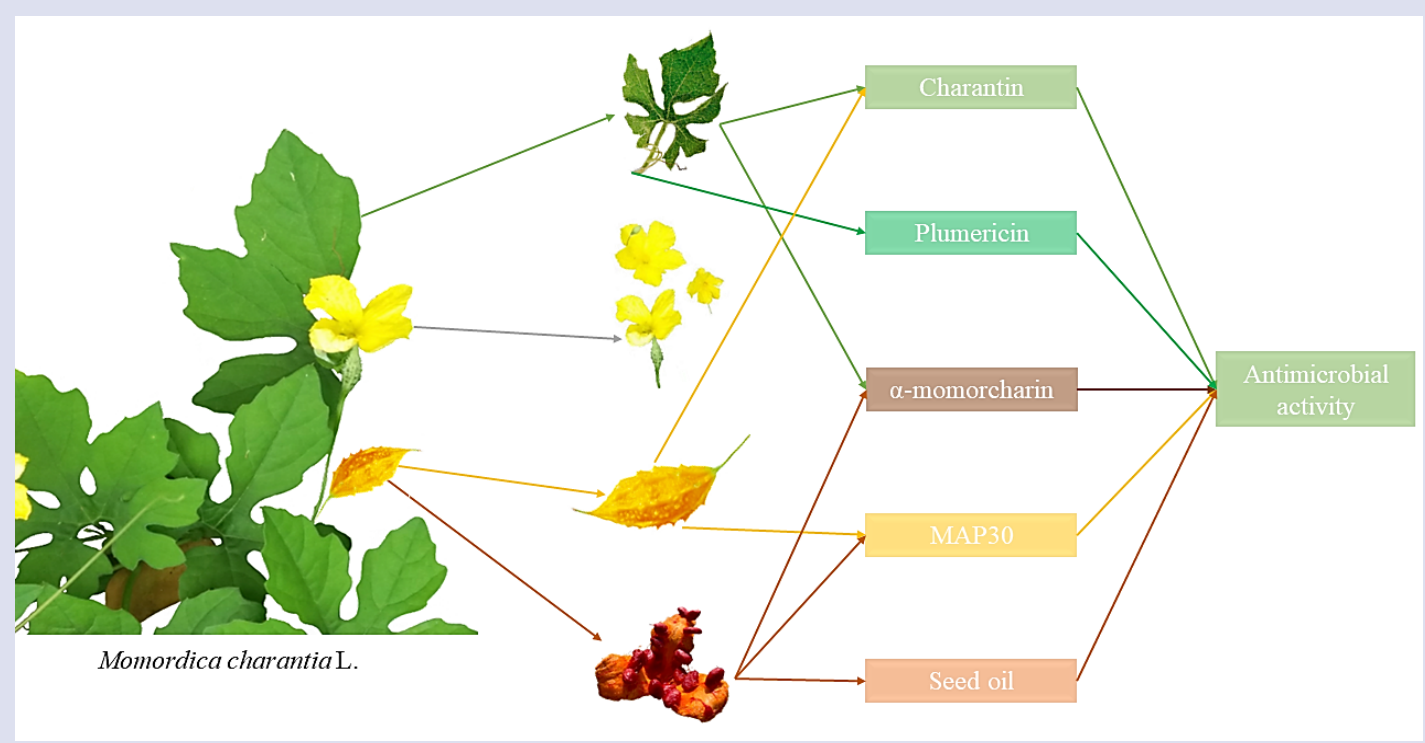

\section{ABOUT AUTHORS}
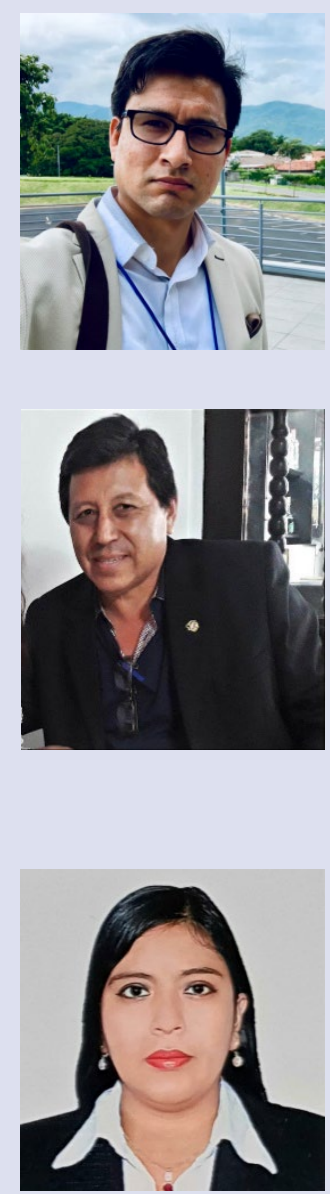

\section{VILLARREAL-LA TORRE VÍCTOR E.}

Affiliation: Departamento de Farmacología, Facultad de Farmacia y Bioquímica, Universidad Nacional de Trujillo

Víctor Eduardo Villarreal La Torre, a Master of Chemical Sciences, holds a degree in Pharmacy from Universidad Nacional de Trujillo (2011). Professor in the Medicinal Chemistry undergraduate program and the Molecular basis of the Action of Xenobiotics postgraduate program at the Universidad Nacional de Trujillo. He currently executes research projects aimed at the discovery of antimicrobial compounds in medicinal plants. Graduate student at Doctoral program in Pharmacy and Biochemistry since 2019.

\section{SAGASTEGUI-GUARNIZ WILLIAM ANTONIO}

Affiliation: Departamento de Farmacología, Facultad de Farmacia y Bioquímica, Universidad Nacional de Trujillo

Professor in the Department of Pharmacology of the Universidad Nacional de Trujillo, Perú since 1993 - to date. I am a graduated in Pharmacy and Biochemistry. Speaker at the graduate program of Universidad Nacional de Trujillo. Has bachelor in pharmaceutical chemistry 1988. Masters in Chemical Sciences, 1999. Doctorate in Biomedical Sciences, graduate program of the Universidad Nacional de Trujillo, 2010. Doctorate studies at Universidade Federal Do Ceará, Brazil, 2015-2018. Currently participates in research projects aimed at the phytochemical characterization of medicinal plants, focusing on antimicrobial activity, resistance to antimicrobials, and antimalarial.

\section{SILVA-CORREA CARMEN ROSA}

Affiliation: Departamento de Farmacología, Facultad de Farmacia y Bioquímica, Universidad Nacional de Trujillo

Professor in the Department of Pharmacology of the Universidad Nacional de Trujillo, holds a degree in Pharmacy and Biochemistry (2011), Master of Chemical Sciences (2017), graduate student at Doctoral program in Biomedical Sciences since 2019. Currently participates in research projects aimed at the phytochemical characterization of medicinal plants, focusing on antimalarial and leishmanicidal activity. In addition, she participates in the evaluation of the wound healing activity of traditional medicinal plants from Peru. 


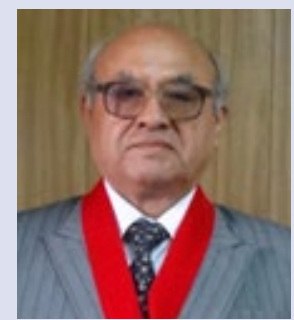

CRUZADO-RAZCO JOSE LIZARDO

Affiliation: Departamento de Farmacología, Facultad de Farmacia y Bioquímica, Universidad Nacional de Trujillo

Professor in the Department of Pharmacology of the Universidad Nacional de Trujillo, holds a degree in Pharmacy and Biochemistry, Master of Physiology and Biophysics, Doctorate studies in Biomedical Sciences. He has participated in research projects on epidemiology of tropical diseases and currently, in research on antimalarial, leishmanicidal and anti-trypanosomal activity of medicinal plants.

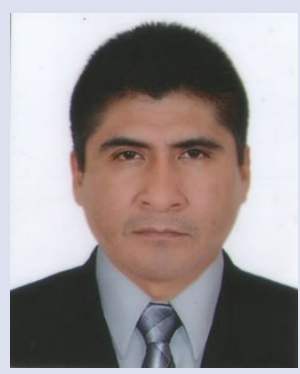

SICHE RAÚL

Affiliation: Facultad de Ciencias Agropecuarias, Universidad Nacional de Trujillo

Graduated in Agroindustrial Engineering - Universidad Nacional del Santa (1996). Master in Economics with a Mention in Business Management - Universidad Nacional de Trujillo (2000). Doctorate in Food Engineering from the Universidade Estadual de Campinas (2008). Post-doctoral studies at the Universidad de Lleida (Spain) in 2012. He founded the scientific journal Scientia Agropecuaria, being until now its Chief Editor. He has been invited to teach courses on experimental designs at the University of Lleida (Spain) and at the University of Sao Paulo (Brazil). It is part of the teaching staff of different doctoral programs at the Universidad Nacional Agraria La Molina and the Universidad Nacional de Trujillo.

Cite this article: Villarreal-La Torre VE, Sagástegui Guarniz W, Silva-Correa C, Cruzado-Razco L, Siche R. Antimicrobial Activity and Chemical Composition of Momordica Charantia: A Review. Pharmacog J. 2020;12(1):213-22. 